\title{
Optimization Models and Algorithms for Operation and Control with Advanced Information Technologies
}

\author{
Junhu Ruan, ${ }^{1,2}$ Xuping Wang, ${ }^{2}$ Chengyan Yue, ${ }^{3}$ Guo Chen, ${ }^{4}$ and Minsoo Kim ${ }^{5}$ \\ ${ }^{1}$ College of Economics and Management, Northwest A\&F University, Yangling, China \\ ${ }^{2}$ Faculty of Management and Economics, Dalian University of Technology, Dalian, China \\ ${ }^{3}$ Department of Applied Economics, University of Minnesota, Minneapolis, MN, USA \\ ${ }^{4}$ School of Electrical and Information Engineering, University of Sydney, Sydney, NSW, Australia \\ ${ }^{5}$ Department of Systems Management and Engineering, Pukyong National University, Busan, Republic of Korea
}

Correspondence should be addressed to Junhu Ruan; rjh@nwsuaf.edu.cn

Received 12 December 2016; Accepted 12 December 2016; Published 25 January 2017

Copyright (C) 2017 Junhu Ruan et al. This is an open access article distributed under the Creative Commons Attribution License, which permits unrestricted use, distribution, and reproduction in any medium, provided the original work is properly cited.

In the literature, lots of optimization models and algorithms have been presented to deal with the operation and control issues in various domains such as intelligent manufacturing, production scheduling, transportation routing, service arrangement, and space exploration. Extant related works have promoted the development of conventional optimization and control theories, and quite a few of them have been widely used in the real world.

With the emergence of recent information technologies including Wireless Sensing Networks, Internet of Things (IOT), Cloud Computing, and Distributed Computing, people can obtain and process far more than what they could, generally within shorter time and with fewer costs. In addition, the application of the information technologies has been bringing about new challenges. One growing challenge is the Big Data problem. Although the Big Data problems and the like may provide chances for people to explore new rules and laws of the universe, they will become catastrophes if not well dealt with.

Thus, people should find new ways and tools in various domains to cope with the emerging challenges. Without exception, extant optimization models and algorithms need to be extended or reformulated for dealing with the reshaped operation and control issues by new information technologies.

This special issue is intended to present optimization models and algorithms for dealing with operation and control issues based on the emerging information technologies. It also aims at promoting the exchanges and interactions between investigators across different fields. We solicit high quality, original research articles as well as review articles focused on models and algorithms for operation and control issues with advanced information technologies.

We received 67 manuscripts in total, made strict preliminary and peer reviews for all the works, and finally accepted 18 papers for the special issue. The included papers can be grouped into five specific categories, that is, (1) models and algorithms with advanced information technologies, (2) healthcare related models and algorithms, (3) models and algorithms of production or delivery, (4) evaluation and classification models, and (5) control models and algorithms.

The first category (models and algorithms with advanced information technologies) includes four papers. The paper "Assessing Partnership Alternatives in an IT Network Employing Analytical Methods" by V. R. Salamat et al. presents an optimization model for the collaborative network building process in the IT industry. In the paper "Exploring an Interactive Value-Adding Data-Driven Model of Consumer Electronics Supply Chain Based on Least Squares Support Vector Machine," X. Wan et al. use the least squares support vector machine to develop a data-driven model of consumer electronics supply chains. The paper "Intelligence in Ecology: How Internet of Things Expands Insights into the Missing $\mathrm{CO}_{2}$ Sink" by W. Wang et al. analyzes the role of the 
Internet of Things (IoT) in monitoring the soil abiotic $\mathrm{CO}_{2}$ uptake and develops an IoT monitoring system. In the work "Two-Phase Algorithm for Optimal Camera Placement," J.W. Ahn et al. develop a two-phase algorithm for the camera placement problem in visual sensor networks.

The second category (healthcare related models and algorithms) includes three papers. The paper "Modeling and Optimization of the Drug Extraction Production Process" by $\mathrm{D}$. He et al. contributes a data-driven iterative learning control method to the drug extraction production process. In the paper "Optimal Scheduling of Doctors Outpatient Departments Based on Patients' Behavior," Z. Ren et al. use the prospect theory to model the patient satisfaction and present a doctor outpatient scheduling model and its plant growth simulation algorithm. The paper "Research on Healthy Anomaly Detection Model Based on Deep Learning from Multiple Time-Series Physiological Signals" by K. Wang et al. uses the convolutional neural network to develop a healthy anomaly detection model.

The third category (models and algorithms of production or delivery) includes six papers. The paper "Impact of Personnel Flexibility on Job Shop Scheduling" by R. Lin et al. contributes a measurement of personnel flexibility as well as a job shop scheduling model with personnel flexibility. The paper "Collaborative Product Design for Tasks Sorting Based on Shortest Delivery" by X. Liang et al. presents a collaborative design task scheduling model. In the work "Identifying and Analyzing Strong Components of an Industrial Network Based on Cycle Degree," Z. Zhang et al. relied on the cycle degree of industrial networks to formulate the concept of circular centrality consisting of the lengths and the numbers of cycles through a vertex. In the paper "Acquisition Pricing and Inventory Decisions on Dual-Source Spare-Part System with Final Production and Remanufacturing," Y. Zhou et al. apply stochastic dynamic programming and recursion algorithm to solve a spare-part inventory problem with two replenishment strategies. The paper "A Recovery Model for Production Scheduling: Combination of Disruption Management and Internet of Things" by Y. Jiang et al. presents a recovery model of production scheduling with the integration of disruption management and Internet of Things. In the paper "A Heuristics-Based Parthenogenetic Algorithm for the VRP with Potential Demands and Time Windows," C. Shi et al. extend the classical vehicle routing problem (VRP) to a VRP with potential demands and time windows (VRP-PDTW) and develop a heuristics-based parthenogenetic algorithm for solving the extended problem.

The fourth category (evaluation and classification models) includes three papers. In the paper "A Novel Evaluation Approach for Tourist Choice of Destination Based on Grey Relation Analysis," X. Guo and Z. Sun apply grey relation analysis into the tourist destination evaluation and show the advantage by the comparison with the Technique for Order Preference by Similarity to Ideal Solution (TOPSIS). The paper "An Optimal Decision Assessment Model Based on the Acceptable Maximum LGD of Commercial Banks and Its Application" by B. Shi et al. presents an optimal credit rating model for customers' credit risk evaluation and credit decision. The paper "A Multiple Classifier Fusion
Algorithm Using Weighted Decision Templates" by A. Mi et al. presents a multiple classifier fusion algorithm using weighted decision templates and shows the advantage by an experimental comparison.

The fifth category (control models and algorithms) includes two papers. In the paper "Optimal Control for Bufferbloat Queue Management Using Indirect Method with Parametric Optimization," A. Radwan et al. integrate an indirect approach with parametric optimization to develop a forward-backward optimal control queue algorithm. The paper "Event-Triggered Faults Tolerant Control for Stochastic Systems with Time Delays" by L. Huang et al. presents an event-triggered scheme of state-feedback controller design for stochastic networked control systems.

\section{Acknowledgments}

We would like to thank all the authors for contributing their works to the special issue and thank all the reviewers for their supporting efforts. Junhu Ruan would like to acknowledge partial supports from China Ministry of Education Social Sciences and Humanities Research Youth Fund Project (no. 16YJC630102); China Postdoctoral Science Foundation (no. 2016M600209); Science and Technology Plan Projects of Yangling Demonstration Zone (no. 2016RKX-04).

\author{
Junhu Ruan \\ Xuping Wang \\ Chengyan Yue \\ Guo Chen \\ Minsoo Kim
}



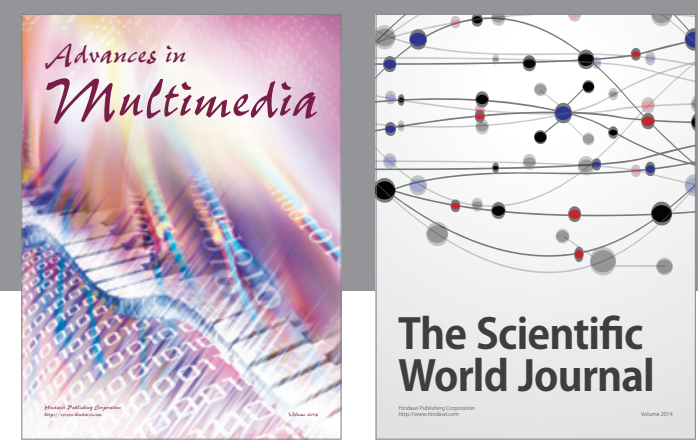

The Scientific World Journal
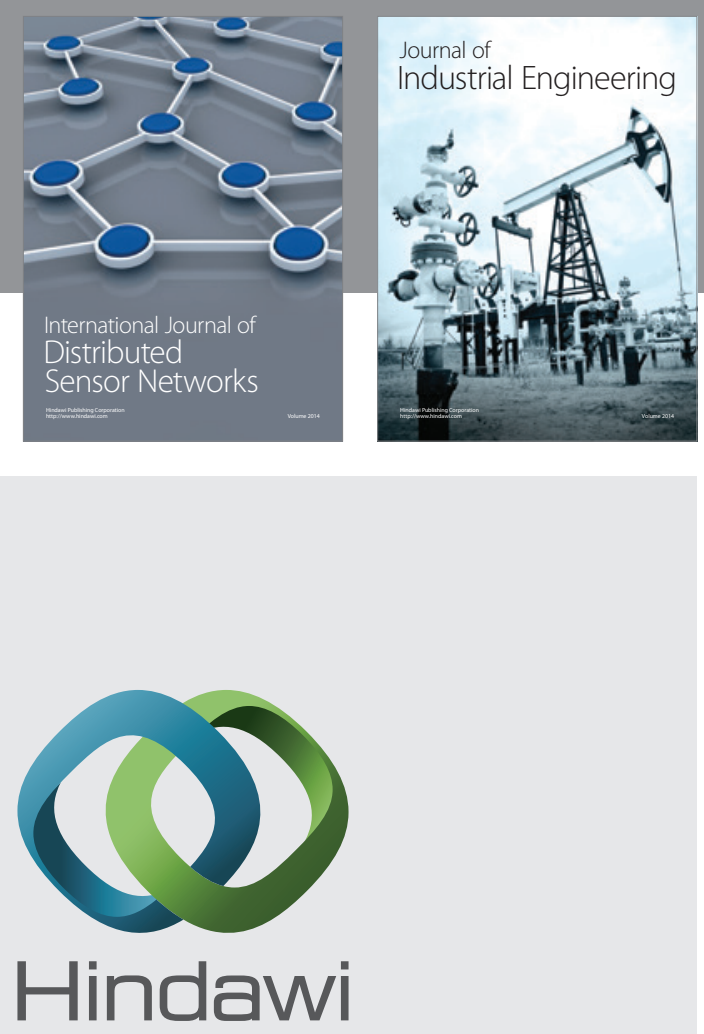

Submit your manuscripts at

https://www.hindawi.com

\section{Computer Networks} and Communications
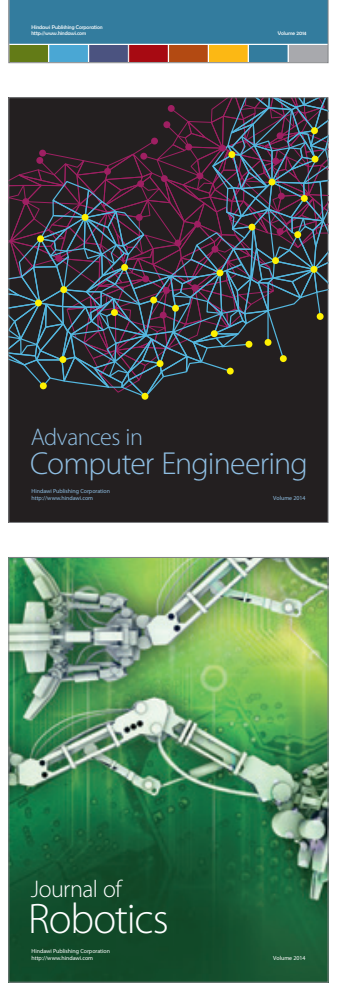
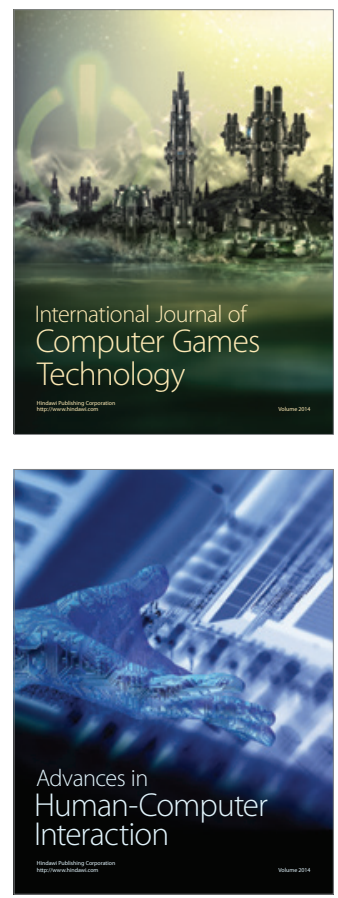
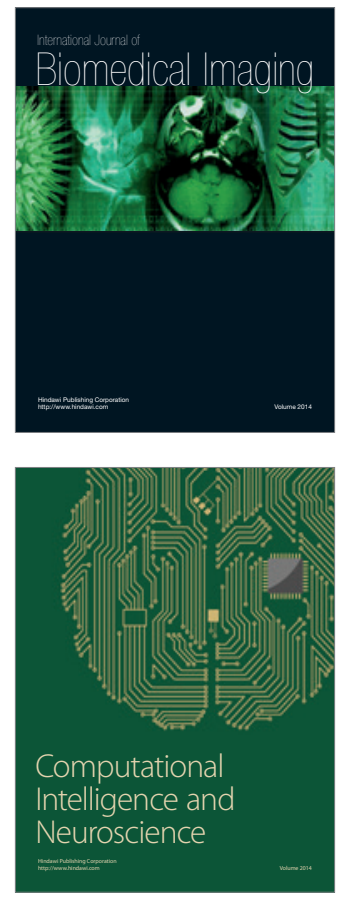
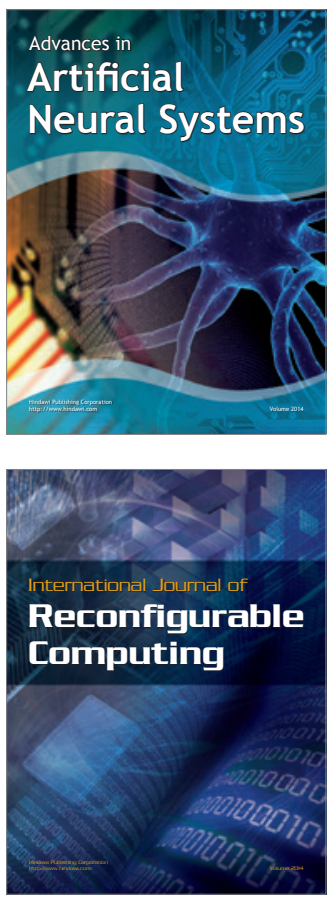
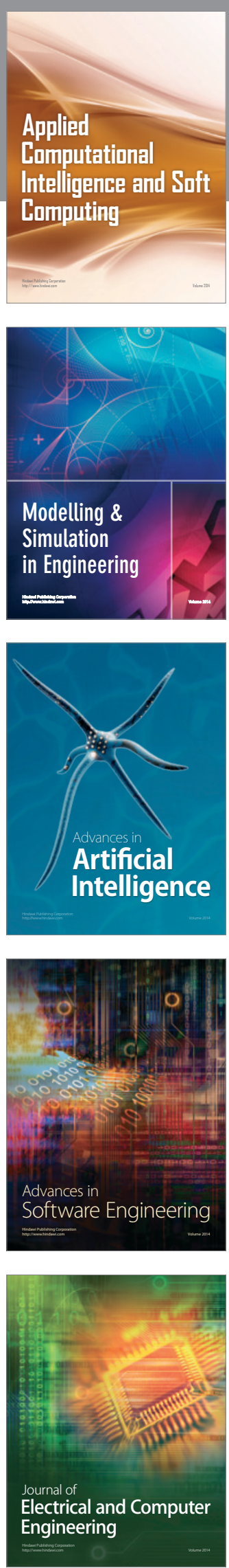